\title{
Anhang 4 \\ Der Brief von Hansteen an Alexander von Humboldt vom 22. Juni 1852
}

Quelle: Staatsbibliothek zu Berlin - Preußischer Kulturbesitz, Nachlass Alexander von Humboldt, Sammlung Darmstaedter 132.30, gr. Kasten 9, Nr. 45, 6 Seiten. ${ }^{336}$

Sternwarte bei Christiania den 22 $2^{\text {sten }}$ Juni 1852.

[Vermerk von Humboldt] 1) Kältepole

2) Schwingungen

Ihre Excellenz!

Der Brief, welcher der Milaneser Lodovico Frapolli ${ }^{337}$ mir in 1846 überbrachte, war mir wahrhaftig eben so unerwartet als erfreulich. Es war wirklich nicht nothwendig, einen so großen Hebel anzubringen, um uns in Bewegung zu setzen, damit wir einen so liebenswürdigen und kenntnißreichen Mann, wie Hrn Frapolli freundlich entgegenkommen sollten. In Betreff dieses Briefes habe ich eine Indiscretion abzubitten. Am Ende des Briefes schreiben Sie: „J'ai eu le plaisir, il y a peu de semaines, de me trouver avec une partie de la charmante Famille de Votre Roi à Rugen. Le jeune Prince m'a paru bien digne d'un père, qui a des sentiments si elevés, et qui ne touchera certainement aux libertés constitutionelles de Votre noble pays.“ Die Königliche Familie war damals hier in Christiania; und da Jedermann erfreut wird, wenn seine gute Eigenschaften anerkannt werden, so übergab ich unserm Statthalter Hrn von Löwenskjold $^{338}$ [sic] eine Abschrift dieser Passage mit der Bitte, sie gelegentlich dem Könige zu communiziren. Hr Löwenskiold hat mir nachher erzählt, daß der König und die Königin sich dadurch sehr erfreut hatten.

Obgleich das Wetter zu der Zeit sehr ungünstig war, so ausführte Hrr. Frapolli unverdroßen eine Reise nach Kongsberg und nach Arendal und kehrte sehr zufrieden zurück. In einem Briefe aus Stockholm (30. Novb. 1846) drückt er seine Zufriedenheit mit seinem Aufenthalte in Norwegen follgermaßen [sic, aus]: „Permettez qu'avant mon départ definitif de cette capitale, je Vous exprime encore une fois mes remercimens les plus vifs pour l'accueil vraiment hospitalier, dont j'ai joui dans votre aimable

336 Herrn Eberhard Knobloch und Herrn Ingo Schwarz, beide von der Alexander-von-HumboldtForschungsstelle an der Berlin-Brandenburgischen Akademie der Wissenschaften, sei herzlich für ihre Hilfe bei der Transkription des Briefes gedankt.

337 Lodovico Frapolli (1815-1878), Offizier, studierte Geologie in Paris. Im Jahre 1848 unternahm er eine geologische Studienreise durch Frankfurt, Deutschland, England und Skandinavien, nahm an den italienischen Befreiungskriegen und 1859 am Krieg Italiens gegen Österreich teil.

338 Severin Løvenskiold (1777-1856), Fabrikbesitzer und Politiker, von 1841 bis zu seinem Lebensende Statthalter von Norwegen. 
Famille à Christiania, et pour toute l'obligeance, que Vous avéz eu envers moi. Le souvenir des personnes, que j'ai eu le bonheur de connaitre à Christiania, le souvenir de la Norvège et de son peuple ne pourront s'effacer sitot de mon esprit. Je partage en cela l'opinion de Votre Roi, qui dans l'audience, qu'il a bien voulu m'accorder, répondit à ma peinture de la Norvège, par les paroles suivantes“: „,,Oh oui, j’aime beaucoup les Norvegiens; c'est un peuple si franc, si loyale, si ami de l'ordre... Leur fierté ne m'inquiète guère; c'est la vigueur de la jeunesse, le sentiment fort d'une nationalité naissante; j’aime cela, je chéris ce peuple“.“ - - Seit dieser Zeit habe ich nichts von dem guten Frapolli gehört, und fürchte sehr, daß sein heftiges Gemüth und starker Freiheitsgefühl Ihm in den unglücklichen Italienischen Unruhen eingezogen haben.

Der Graf von Beust, ${ }^{339}$ welcher mir einen zweiten sehr schmeichelhaften Brief von Ihnen überbrachte, reiste den folgenden Tag mit seiner Gemahlin nach Kongsberg und Modum und wird bald zurück erwartet. Ich war nicht zu Hause, da er die Sternwarte besuchte, traf Ihm aber den folgenden Vormittag ein Augenblick in dem zoologischen Cabinette, wo H[er]r Professor Boeck ${ }^{340} \mathrm{ihm}$ die Präparate und Sammlungen vorzeigte, und hatte bloß Gelegenheit ein Paar Worte mit Ihm zu sprechen. Er versprach nach seiner Zurückkunft die Sternwarte zu besuchen. Er hat ein sehr ungünstiges Wetter zu seiner Reise erhalten.

[S. 2] Ich habe oben gesagt, daß der Brief, welcher Her[r] Frapolli überbrachte, mir ebenso erfreulich als unerwartet war. Ich muß mich darüber näher erklären, und hoffe, daß Sie meine freimüthige Aeußerungen mit der bekannten Humanität aufnehmen werden. Ich hatte mir in einer Reihe von 30 Jahren, die Ueberzeugung in den Kopf gesetzt, daß Sie einigen Unwillen gegen mich hegten. Ueberall nämlich wo Sie in Gilberts und Poggendorffs Annalen vom Magnetismus der Erde sich äußerten, wurde niemals mein Name genannt, wo Sie sonnst die unbedeutendsten Beobachtungen Anderer, und bisweilen $\underline{\text { lose }}^{341}$ Hypothesen von A. T. Kupffer erwähnten. Ein Paar Mal wurde obiter hin mein Name mit einen kleinen Tadel berührt. Eine Polemik in Kosmos I. S. 432-435 342 glaubte ich auf mich gerichtet, weil ich in Poggendorffs Annalen Bd. 28, S. 474-479343 in chronologischer Ordnung die Bestrebungen um

339 Graf Friedrich Ferdinand von Beust (1809-1886), Staatsmann im Dienste von Sachsen und seit 1866 von Österreich-Ungarn, ab 1849 Außenminister und ab 1858 zusätzlich Vorsitzender des Gesamtministeriums des Königreichs Sachsen.

340 Christian Peter Bianco Boeck (1798-1877), seit 1828 Lektor für Veterinärmedizin an der Universität in Christiania, 1840 Professor für Physiologie und vergleichende Anatomie.

341 Man kann nicht deutlich erkennen, wer das Wort „lose“ unterstrichen hat, möglicherweise weder Hansteen noch Humboldt.

342 „Folgendes ist der historische Hergang der Auffindung des Gesetzes von der (im allgemeinen) mit der magnetischen Breite zunehmenden Intensität der Kräfte“ (Humboldt 1845-1862: 1, S. 432).

343 „Ueber das magnetische Intensitätssystem der Erde“ (Hansteen 1833a). Im selben Band der „Annalen der Physik und Chemie“ erschien auch Poggendorffs Übersetzung von Gauß’ bahnbrechender Arbeit „Die Intensität der erdmagnetischen Kraft zurückgeführt auf absolutes Maaß“ (Gauß 1833). 
das Gesetz der magnetischen Intensität zu entdecken angeführt habe, und dabei die Namen Mallet ${ }^{344}$, Lamanon, ${ }^{345}$ de Rossel, v. Humboldt, Sabine u. s. w. bis Admiral Lütke erwähnt habe. In dem nämlichen Bande S. 439 stehet: „Der denkwürdige Zusammenhang zwischen der Krümmung der magnetischen Linien und der Krümmung meiner Isothermen ist zuerst von Sir David Brewster aufgefunden worden. Dieser berühmte Physiker nimmt in der nördlichen Erd=Hälfte zwei Kältepole an, einen Amerikanischen und einen Asiatischen u. s. w. ${ }^{[๘] 346}$

[Anmerkung von Humboldt am rechten Rand des Briefes] Wem die Kältepole gehören? ${ }^{347}$

In der Vorrede meiner „Untersuch. über den Magn. der Erde“ S. XI-XII welcher gedruckt ist in 1818 aber geschrieben ${\underline{1813^{348}}}^{3 e r d e n ~ S i e ~ f o l g e n d e ~ S t e l l e ~ f i n d e n: ~ „ E s ~}$ spricht die Erde mittelst der stummen Sprache der Magnetnadel die Bewegungen in ihrem Inneren aus, und verstünden wir des Polarlichtes Flammenschrift recht zu deuten, so würde sie für uns nicht weniger lehrreich seyn. ${ }^{\star}$ )

[Anmerkung von Hansteen am rechten Rand des Briefes] $\left(^{\star}\right)$ Diesen Ausdruck hat, so viel ich erinnere Brewster, ${ }^{349}$ als geschmackloß erklärt. Ist dieses so, so hat er doch die Stelle gelesen.

[Fortsetzung des Absatzes] Der Zusammenhang der Meteorologie mit dem Polarlichte, folglich mit den magnetischen Kräften, springt in die Augen; ebenso merkwürdig ist

344 Frederik Mallet war zuerst Observator an der Sternwarte in Uppsala, seit 1773 Professor der Geometrie an der Universität Uppsala.

345 Robert Paul de Lamanon machte die magnetischen Beobachtungen auf der im Jahre 1785 beginnenden La Pérouse-Expedition.

346 Vergleiche auch eine entsprechende Stelle in Humboldts „Kosmos“: „Der denkwürdige Zusammenhang zwischen der Krümmung der magnetischen Linien und der Krümmung meiner Isothermen ist zuerst von Sir David Brewster aufgefunden worden; s. Transactions of the Royal Society of Edinburgh Vol. IX. 1821 p. 318 und Treatise on Magnetism 1837 p. 42, 44, 47 und 268. Dieser berühmte Physiker nimmt in der nördlichen Erdhälfte zwei Kältepole (poles of maximum cold) an, einen amerikanischen ( $\operatorname{Br}\left[\right.$ eite] $73^{\circ}$, Länge $102^{\circ}$ West, nahe bei Cap Walter) und einen asiatischen $\left(\mathrm{Br}\left[\right.\right.$ eite] $73^{\circ}$, Länge $\left.78^{\circ} \mathrm{Ost}\right)$; daraus entstehen nach ihm zwei Wärme $=$ und zwei Kälte=Meridiane, d. h. Meridiane der größten Wärme und Kälte“ (Humboldt 1845-1862: 1, S. 439).

347 Diese Diskussion hat Hansteen auch noch 1860 verfolgt. In seinem Aufastz „Das magnetische System der Erde“ ist zu ihr zu lesen: „In der Vorrede meiner «Untersuchungen über den Magnetismus der Erde» (geschrieben 1813) habe ich mich darüber folgendermaassen geäussert: «Der Zusammenhang der Meteorologie mit dem Polarlichte, folglich mit den magnetischen Kräften, springt in die Augen [...]. Vielleicht möchte ein tieferes Studium der magnetischen Kräfte der Erde über diese dunkeln Gegenstände das gehörige Licht verbreiten. Diese Bemerkungen haben später Herrn Dr. Brewster bewogen, diese Regionen den Namen «Kältepole» zu geben» “ (Hansteen 1860, S. 67-68).

348 Möglicherweise wurde „1813“ von Humboldt unterstrichen.

$349 \mathrm{Zu}$ Brewster siehe Kap. 2.6.3. Mit ganz ähnlichen Worten wie gegenüber Humboldt hatte Hansteen seine Kritik an Brewster schon viel früher, im Jahre 1833, Gauß mitgeteilt. In einem undatierten Brief verwendete er den Ausdruck „abgeschmackt“ (siehe Brief Nr. 5, S. 6). Brewsters Kritik bezog sich allerdings auf die Aussage „ «The mathematicians in Europe» (says Mr Hansteen, with more truth than taste)“ und nicht, wie man hier vermuten möchte, auf die „Flammenschrift“ des Polarlichtes (Brewster 1820/1821, S. 127). 
die Gleichheit zwischen Humboldts isothermischen Linien und den magnetischen Neigungslinien. Wer hat noch die Kälte Sibiriens, Grönlands und des Feuerlandes zu erklären vermocht? Wer die sonderbaren Veränderungen der Polarklimate, oder Cooks Bemerkungen über die verschiedenen Abstände der festen Eisflächen vom Südpole im stillen und in dem Atlantischen? Sie sehen hier eine deutliche Anspielung an 4 sogenannte Kältepole, in Nordamerika, in Sibirien, unter Neuholland und südwestlich vom Feuerlande, kurz in den 4 Meridianen wo die magnetische Neigung und Intensität auf dem selbigen Breiten=Parallel ein Maximum hat, oder nach meinem Sprachgebrauche ein Magnetpol liegt. In der handschriftlichen Vorrede war dieses noch etwas ausführlicher ausgedrückt; und da ich zu der Zeit mit Sir D. Brewster in Correspondance war, und ihm meine Untersuchungen und Neigungs- sammt Intensitätscharten mittheilte, so war seine Erfindung sehr leicht. Doch gebührt

[S. 3] Ihm die Ehre der Erfindung des Namens Kältepole (poles of maximum cold), welchen ich unpassend finde; wie überall der häufige Misbrauch des Namens „Pol“.

Ich fing im Jahre 1807 in meinem $23^{\text {sten }}$ Jahre an Materialien $\mathrm{zu}$ einer Untersuchung über den Erdmagnetismus zu sammeln, und dieß unter den ungünstigsten Umständen in der kleinen Stadt Friedrichsburg, 4²/2 Meilen von Kopenhagen, und gieng jeden Sonabend zu Fuß nach Kopenhagen, um Reisebeschreibungen und andere Bücher aus dem königl. Bibliotheke zu holen; zeichnete Karten, und fortsezte unablaßend diese Arbeit bis 1813, da die Abhandlung die Preismedaille der Gesellschaft der Wissenschaften in Kopenhagen gewann. Ich gestehe, daß ich damals ein mathematischer Novize war, und daß die Arbeit von dieser Seite unreif ist. In Christiania fortsezte ich die Arbeit und die weitläuftigen Rechnungen bis 1818, da der König Carl Johan ${ }^{350}$ sich entschließ, meine Arbeit drucken zu lassen. Von dieser Zeit fing ich selbst an Beobachtungen anzustellen, und Freunde aufzumuntern auf Ihre Reisen mit meinem Apparate Intensitätsbeobachtungen anzustellen. Ich entdeckte in 1821-1822 durch 5 bis 7 tägliche Oscillationsbeobachtungen mit einem horizontal schwingenden Cylinder die tägliche reguläre Variation der horizontalen Intensität (Minimum Vormittags um 10 Uhr, Maximum Nachmittags im Sommer 6-7 Uhr im Winter um 4 Uhr). Durch meine eigene und meiner Freunde Beobachtungen publicirte ich die erste Charte über isodynamische Linien der horizontalen Intensität, und nachher auch über die totale Intensität, nach meiner Rückkunft von Sibirien endlich die erste Universalcharte der totalen Intensität (Verkleinert in Poggendorfs Annalen Bd. 28). Durch meine Versuche und Calcüle in „Magnet. der Erde“, hatte ich doch überzeugend erwiesen: daß die magnetische Molecular-Anziehung sich umgekehrt verhällt wie die Quadrate der Abstände (Magn. der Erde S. 136); und durch eine Reihen=Entwickelung daß die Totalwirkung eines Linear-Magneten (ein prismatischer Magnet mit verschwindender Dicke) durch eine Reihe ausgedrückt werden kann aus folgender Form $\mathrm{I}=\frac{\alpha}{a^{3}}+\frac{\beta}{a^{5}}+\frac{\gamma}{a^{7}}+\ldots$ wo $a$ der Abstand des angezogenen Punkt vom Mittelpunkts des Magneten, $\alpha, \beta, y$. con-

350 Ab 1818 König von Schweden (Karl XIV.) und König von Norwegen (Karl III.). 
stante Größen, die von der Vertheilung des Magnetismus im Stabe abhängen; folglich wenn $a$ sehr groß ist, umgekehrt wie die $3^{\text {tte }}$ Potenz des Abstandes. Dieses sind dieselbigen Gesetze, die Gauß, ohne den Stab als lineair vorauszusetzen, auf einer eleganteren Weiße demonstrirt hat. - Da ich in 1839 Göttingen besuchte, sagte mir Gauß, der durch Schumacher mit mir in Correspondance gekommen war: „Sie sind sehr daran Schuld, daß ich mich zu dem Magnetismus der Erde hingewendet habe“.`

[Anmerkung von Humboldt am rechten Rand des Briefes, besteht aus zwei Teilen] * Wie wenig ist solchen Aeusserungen zu glauben. Als ich gleich nach Erschein[en] der Theorie an Gauss schrieb ich hoffe sein Wohnen bei [mir] + Beschäft[igung] mit meiner Boussole v. Gambey habe ihn [angeregt] ${ }^{351}$ antwortete er erheitert Er habe das schon früher im Kopf gehabt!! ${ }^{352}$

[Fortsetzung des Absatzes] Wenn ich auch nichts mehr in meinem Leben ausgerichtet hätte, als dieß letzte, so würde ich ganz zufrieden seyn, und sagen: „non frustra vixisse videor.“ Ich hatte schon 1822 auf eine absolute Bestimmung

[S. 4] der magnetischen Intensität gedacht, und die Methode gefunden durch Oscillationen eines Magnetstabes, und durch Anwendung desselbigen nachher als Ablenkungsstab das Verhältniß der Intensität zu der Schwere zu bestimmen. Theils aber hatten wir keinen Instrumentenmacher, der Apparate für so feine Abweichungen machen könnte; theils war ich nicht wie Gauß auf die einfache Idee gekommen, die Constanten $\beta$ und $y$ durch Ablenkungen in verschiedene Abstände zu eliminiren. Da Gauß später sich mit derselbigen Aufgabe beschäftigte, mittheilte er mir seine Gedanken, welche etwas verschieden von meinem Plane waren, und ich communicirte Ihm meinen Vorschlag welchen er auch folgte mit der obenerwähnten nothwendigen Verbesserung, und mit größeren und bequemeren Instrumenten. Ich kann folglich ebenso wie Sie (Kosmos I. S. 435) mutatis mutandis sagen: „daß man einigen Werth auf das legt, womit man sich 30 Jahrelang ununterbrochen beim Arbeitstische und auf Reisen in Sibirischer Kälte, wo das Quecksilber gefriert, beschäftigt hat.“ - Aber, um nach dieser langen Abschweifung auf den Anfang zurückzukommen, es ist mir außerordentlich erfreulich aus Ihren zwei Briefen zu sehen, daß meine Vorstellung falsch war.

Gauß verstehet bei magnetische Pole die zwei Punkte auf der Oberfläche der Erde, wo die magnetische Resultante des Erdkörpers vertikal ist, also mit der Richtung der Schwere zusammenfällt; ich die Regionen, wo die magnetische Kraft ein Maximum hat. Gaußens Pole haben folglich bloß eine mathematische, meine eine physische Bedeutung. Die Frage, ob die Erde 2 oder 4 Magnetpole habe, ist also bloß ein leerer Wortstreit. Wenn die Erde nach meinem Sprachgebrauche 2 Magnetpole in jeder Halbkugel hat, so ist es klar, daß der Gaußische Pol irgendwo zwischen diese beide

351 Im Original stehen zwei gezeichnete Quadrate mit einem Punkt als Hinweis auf die Fortsetzung der Anmerkung.

352 Siehe hierzu (Reich 2011). 
liegen muß, und wenn sie von verschiedener Intensität sind, näher an den intensiveren. ${ }^{353}$ In „Magnet[ismus] der Erde“ S. 375 habe ich durch Rechnung nach meinen Elementen gefunden, daß dieser Gaußische Nordpol in Breite 735’, Länge $291^{\circ}$ Ost Ferro liegen sollte, welches nicht viel von der Wahrheit abweichen dürfte. - Diese Gaußische Benennung scheint mir unbequem. Wenn die Erde ihre Form veränderte, ohne daß die magnetische Resultante ihre Richtung änderte, so würde der Gaußische Pol seine Lage verändern.

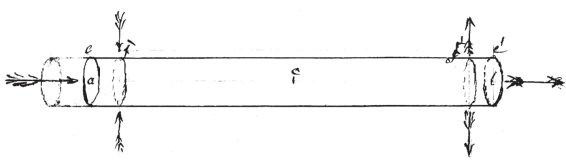

Ein cylindrischer, auf der Achse senkrecht abgeschnittener Magnetstab würde nach der Gaußischen Benennung $1^{\text {stens }}$ zwei Pole haben in den beiden Mittelpunkten $a$ und $b$ der Endflächen, und $2^{\text {tens }}$ zwei Polarflächen senkrecht auf der Cylinderachse in der Nähe der Endflächen, wo die mit der Achse $a b$ parallele Resultante des kleineren aber intensiveren Stücks zwischen $e$ und $f$ ist gleich der Resultante des längeren aber schwächeren und entfernteren Stücks von $f^{\prime}$ bis $e^{\prime}$. Wenn die Endflächen nicht senkrecht auf die Achse $a b$ wären, so würde alles dieses sich änderen. Hiebei wird doch der Begriff „Magnetpol“, wobei man sich eine physische Eigenschaft des

[S. 5] Körpers denken muß, ganz verwirrt. ${ }^{354}$

Ich erlaube mir noch einige Bemerkungen über die Requisiten einer guten relativen magnetischen Intensitätsbestimmung. 1) Die Nadel muß unter der Reise einen unveränderlichen Magnetismus haben. Da dieses aber bei neu gestrichenen Nadeln niemals der Fall ist, wenn man nicht durch mehrmaliges Untertauchen in Wasser von einer höheren Temperatur, als die für welche sie unter der Reise ausgesetzt werden können, die unhaltbare Intensität verloren haben. Da man doch darüber nicht ganz sicher sein kann, muß man ihre Schwingungen vor und auf der Reise auf demselbigen Punkt beobachten, um wenigstens einen kleinen Verlust interpoliren zu können. 2) Man muß den Schwingungsbogen bei Anfang der Beobachtungen notiren, und wenigstens bei welcher Schwingung der Bogen auf die Hälfte abgenommen hat, um durch Rechnung die ganze Schwingungszeit auf der Zeit eben soviel Schwingungen in verschwindende Bögen zu bestimmen. Je größer der Anfangsbogen ist, desto nothwendiger ist diese Reduction. Beobachtet man z. B. auf einem Punkt mit einem Anfangsbogen von $40^{\circ}$, auf einen andern mit einem Bogen von $30^{\circ}$, auf einem Punkt

353 Die ersten Zeilen dieses Absatzes bis zu dieser Stelle sind von Humboldt auf dem Seitenrand durch einen Doppelstrisch hervorgehoben.

354 In dem Brief von Hansteen an Gauß vom 4. August 1840 (Brief Nr. 10) wurde schon vieles von dem erwähnt, was Hansteen jetzt auch Alexander von Humboldt wissen ließ. Dort findet sich auch eine ganz ähnliche Zeichnung. 
100 Schwingungen, auf einem andern 120, so sind diese Beobachtungen nicht comparable ohne die besagte Reduction. 3) Man muß den Einfluß der Temperatur auf das Moment der Nadel untersucht haben, da diese sehr stark ist. Ich machte z. B. auf verschiedene Punkte auf der Hin- und Rückreise durch Sibirien Beobachtungen unter sehr verschiedenen Temperaturen und fand

\begin{tabular}{|c|c|c|c|c|}
\hline St. Petersburg & 1828 Juli 6 & $\begin{array}{c}\text { Temperat[ur] } \\
\text { der Nadel } \\
+24^{\circ}, 4 \mathrm{R}^{355}\end{array}$ & $\begin{array}{c}\text { Zeit von } 300 \\
\text { Schw[ingungen] } \\
802,02\end{array}$ & $\begin{array}{c}\text { Reducirt } \\
\text { auf }+7^{\circ}, 5 \\
798,55\end{array}$ \\
\hline Bot[anischer] & 1830 Mai 3 & +7.2 & 800,93 & 801,02 \\
\hline \multicolumn{5}{|l|}{ Garten } \\
\hline Tomsk & 1829 Jan. 14 & $-20^{\circ} .8$ & 731,66 & 736,41 \\
\hline IOHSK & 1829 Sept. 10 & +15.3 & 739,36 & 737,56 \\
\hline \multirow{2}{*}{ Krasnojarsk } & 1829 Jan. 23 & -20.8 & 724,09 & 728,77 \\
\hline & 1829 Aug 8 & +19.9 & 732,13 & 729,42 \\
\hline Tschernoi- & 1830 Febr 12 & -16.9 & 670,67 & 674,53 \\
\hline Jarr & 1830 März 1 & -1.75 & 672,99 & 674,26 \\
\hline
\end{tabular}

[Dieser Absatz steht auf dem rechten Rand der Tabelle] In Tomsk war der Unterschied der Temperatur in dem Kasten $=36^{\circ}, 1 \mathrm{R}$. in Krasnojarsk $=40^{\circ}, 7 \mathrm{R} \cdot .^{356}$ Die Differenz der unreducirten Zeit von 300 Schwingungen war auf dem ersten Punkt $=7^{\prime \prime} .7$, auf dem zweiten $=8$ "“.04, und doch stimmten die reducirten Schwingungszeiten bis auf 1 "“.15 und 0 "“.65.

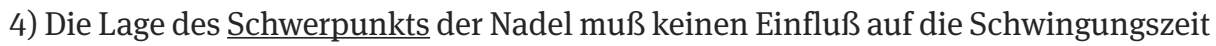
haben. Da der Schwerpunkt der Neigungsnadel niemals vollkommen genau in der Achse der Zapfen liegt, welches die Angabe der Nadel in den 4 verschiedenen Lagen zeigt, und auch von keiner menschlichen Geschicklichkeit verlangt werden kann, und dieser Schwerpunkt in den verschiedenen Neigungen verschiedene Lage gegen die durch die Umdrehungsachse gehende Vertikalfläche hat, so hat dieser einen veränderlichen Einfluß auf die Schwingungszeit. Deswegen ist auch der Vorschlag, die Neigung durch Beobachtung der Schwingungszeit in magnetisch Azimuth $0^{\circ}$ und $90^{\circ}$ verwerflich. 5) Die Nadel darf nicht unter der ganzen Reihe der Beobachtungen in Berührung kommen mit Eisen oder Magnet. Folg-

[S. 6] lich ist die Methode des Capit. Sabine auf der Pendelexpedition, die Nadeln paarweise mit Ankern von weichem Eisen aufzubewahren, nicht zu billigen. Bei jeder solchen Berührung verändert sich das Moment der Nadel mehr oder weniger, und kann nicht interpolirt werden. 6) Der Gang der Uhr muß angegeben werden.

Auf meiner Reise durch Rusland und Sibirien wurden alle diese Vorsichtsmaaßregeln und Reductionen in Acht genommen, ebenso auf der Beobachtungsreise meiner

$35524,4^{\circ}$ nach der Réaumur-Temperaturskala entsprechen $30,5^{\circ}$ Celsius. $35640,7^{\circ}$ nach der Réaumur-Temperaturskala entsprechen 50,9 $9^{\circ}$ Celsius. 
Freunde Keilhau, Boeck und Abel durch Deutschland, Tyrol, Schweitz nach Italien. ${ }^{357}$ Auf allen früheren Reisen wurden mehrere dieser Berichtigungen, und auf etlichen alle, negligirt. Mr. de Rossel hat doch die Elongation der Nadel (den Schwingungsbogen) bei jeder $10^{\text {ten }}$ Schwingung aufgezeichnet, gewöhnlich mit $30^{\circ}$ angefangen, und die Reduction auf verschwindende Bögen berechnet. Wohl ist diese Reduction erst nach der Rückkehr nach Frankreich ausgeführt; wenn aber ein Beobachter auf solche nothwendige Reductionen aufmerksam ist, und die dazu nothwendige Data aufzeichnet, so ist es nicht wahrscheinlich, daß er sogleich bei Vollendung einer Beobachtung versäumt haben sollte zu bemerken, welche Veränderung der Schwingungszeit eingetreten wäre. Betrachtet man bloß folgende rohe unreducirte Zeit von 100 Schwingungen seiner flachen Nadel (l'aiguille plate), so ist der Unterschied zwischen Amboina und Van Diemens Land zu groß, um übersehen zu werden; eine solche Gleichgültigkeit kann man einem Beobachter, der auf die feineren Reductionen im voraus aufmerksam ist, nicht zutrauen.

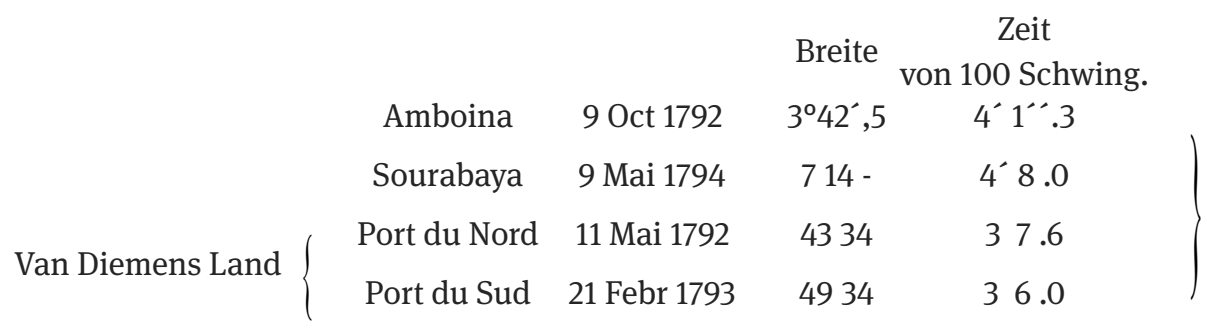

In 285 Tagen zwischen $11^{\text {ten }}$ Mai 1791 und 21 Febr[uar] 1793 hatte die aiguille plate folglich nichts von ihrer Intensität verlohren; und vom Amboina bis Van Diemens Land ist die Zeit von 100 Schwingungen um 54" bis 55"“ abgenommen.

Den 28 ${ }^{\text {ten }}$ Juli. In der seit Anfang dieses Briefes verflossenen Zeit ist der Hr. Graf von Beust zurückgekehrt und nach Schweden abgereist. Ich habe mir die Freiheit genommen Ihm das Resultat 14jähriger meteorologischer Beobachtungen auf der hiesigen Sternwarte für Ihre Excellenz mitzugeben, ein Abdruck von einer kleinen Abhandlung, in den Verhandlungen der Schwed[ischen] Akademie der Wissenschaften, worin die tägliche regulaire Variation der Temperatur und des Luftdrucks durch stündliche Beobachtungen Tag und Nacht ermittelt ist. - Ich bitte nun noch einmal diese freien Bemerkungen nicht als eine Polemik aufzunehmen, in dem ich mich mit der größten Hochachtung unterzeichne

Ihro Excellenz ehrerbietigster Diener Chr. Hansteen. 


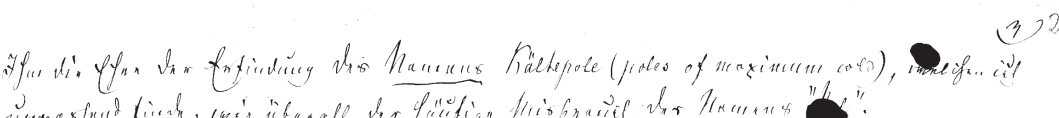

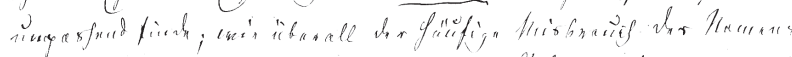

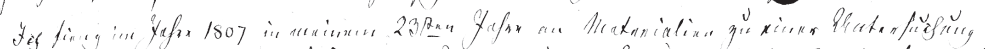

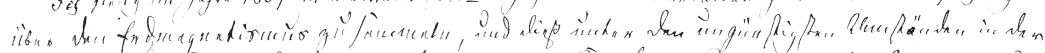

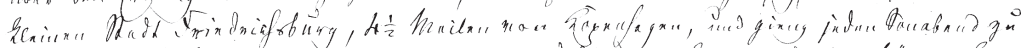

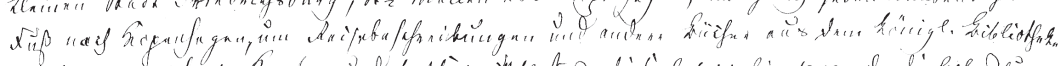

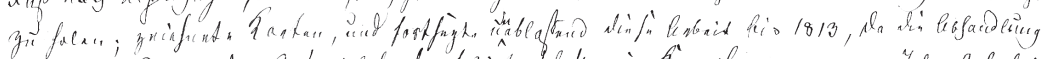

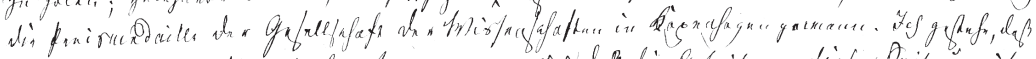

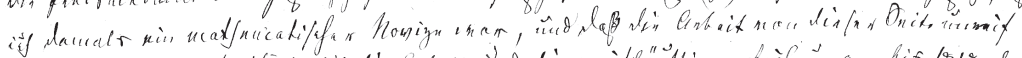

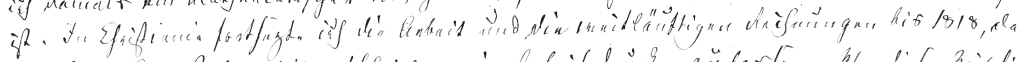

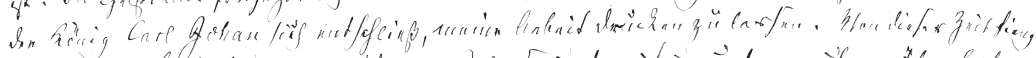

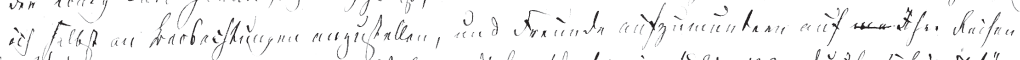

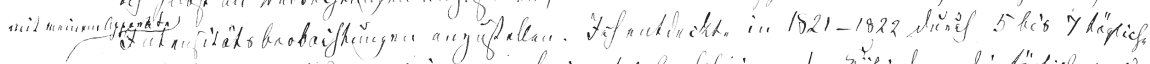

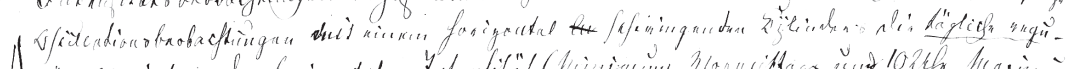

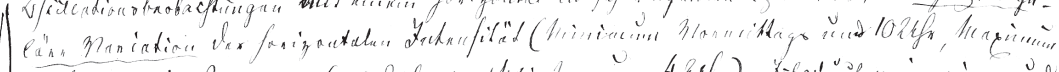

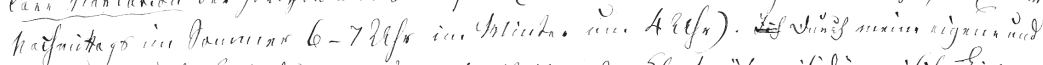

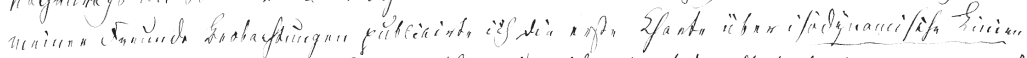

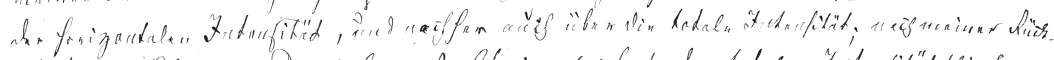

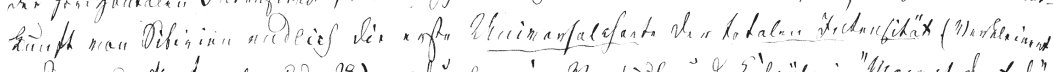

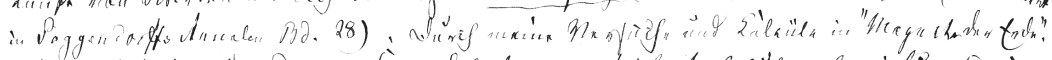

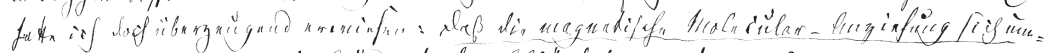

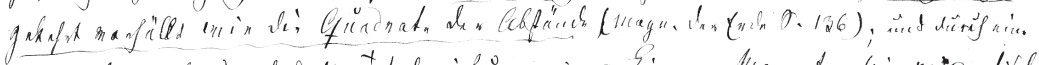

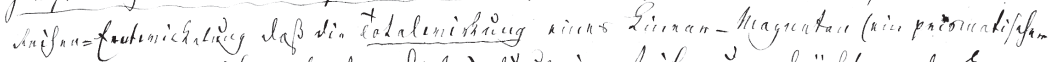

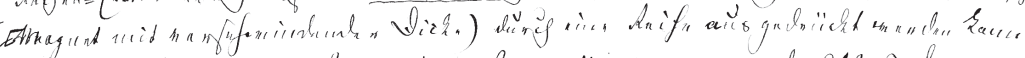

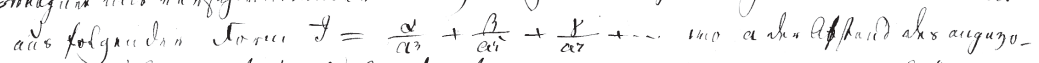

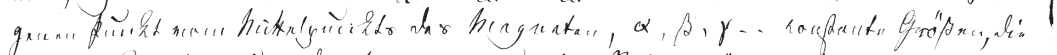

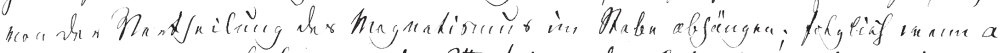

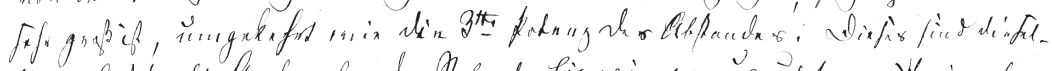

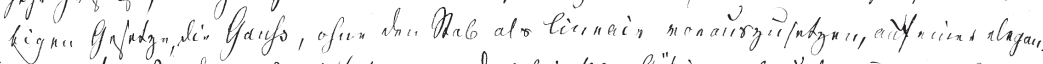

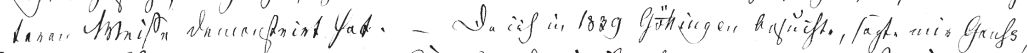

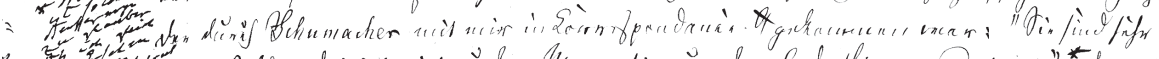

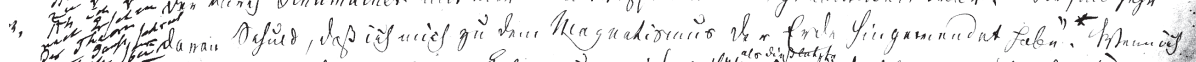

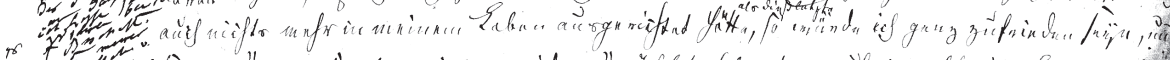

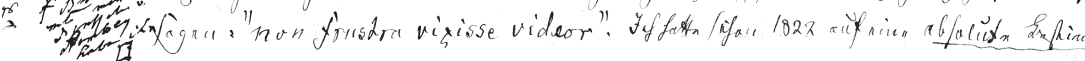

Abb. 44: Die dritte Seite des Briefes von Christopher Hansteen an Alexander von Humboldt vom 22. Juni 1852 (Sternwarte bei Christiania) mit Anmerkungen von Humboldt. Staatsbibliothek zu Berlin - Preußischer Kulturbesitz, Nachlass Alexander von Humboldt, Sammlung Darmstaedter 132.30, gr. Kasten 9, Nr. 45, BI. 2r. 